\title{
Coronavirus Infection in Children
}

\section{Bannova Svetlana Leonidovna, Puzyrev Viktor Gennadievich}

Federal State Budgetary Educational Institution of Higher Education "St. Petersburg State Pediatric Medical University" of the Ministry of Health of the Russian Federation.

\begin{abstract}
SARS-COV-2 is a highly contagious anthroponotic infection. The relevance of the study of the disease is due to the wide prevalence of the pandemic worldwide to the extent that it affects all age groups and populations, the lack of long-term and intense immunity, and high mortality, mainly among adults.Children are also susceptible to SARS-COV-2 infection, but this age group is more likely to be asymptomatic. This article summarizes the main trends in clinical manifestations and treatment of coronavirus infection in children available in current moment.
\end{abstract}

Keywords: Covid19- Treatment- Clinical manifestations- Examination

Asian Pac Environ Cancer, 3 (Suppl 1), 5-8

\section{Introduction}

Children are also susceptible to SARS-COV-2 infection, but this age group is more likely to be asymptomatic. According to statistics, children account for about $7.6 \%$ of registered cases in the Russian Federation, of which the first place is given to family centers. At the present stage, children may play a huge epidemiological role in the spread of the virus, as in the absence of clinical signs of respiratory disease, the maximum periods of fecal release have not yet been fully studied. In addition, the pathogenesis and clinical picture in children is not fully understood, especially in the presence of severe concomitant pathology from risk groups. SARS-COV-2 is a "new" infection for which at the current moment no specific effective antiviral therapy schemes have been found both in adults and children.

The purpose of this review is to summarize the main trends in clinical manifestations and treatment of coronavirus infection in children.

Mechanism of transmission: aerosol as well as alimentary. Transmission pathway - airborne, droplet and contact pathway, as well as faecal-oral [1-2-3-4-5].

At present, there is no conclusive evidence of vertical mother-to-child transmission (the virus is not detected in amniotic fluid and vaginal secretion), nor has there been any evidence of secretion of the causative agent with breast milk. However, there is evidence that in cases of
Submission Date: 06/15/2020Ａcceptance Date: 07/07/2020

COVID-19 infection, the risk of early delivery, low birth weight relative to gestational age increases [6].

SARSCoV-2 susceptibility is universal. At present, adults are mainly ill, and children account for 1 to $5 \%$ [1-2-7]. Children carry the disease in asymptomatic and mild forms [2-8]. Factors such as congenital immune response feature, favourable condition of respiratory mucosa, maturation and functioning of angiotensinconverting enzyme, fewer chronic diseases, fewer international travel, communication and movements, absence of harmful habits may explain the resistance of children to SARS-CoV-2.

However, the risk of development of the severe course of COVID-19 is typical for children from risk groups (new-borns and children of early age; children with unfavourable pre-morbid background, especially congenital malformations, diseases of cardiovascular system, respiratory organs, diabetes mellitus, malignant neoplasms, Kawasaki disease; children with immunodeficiency conditions of different genesis; children with co-infection, especially respiratory syncytial at early age) [1-2].

The incubation period is from 2 to 14 days (5-7 days on average), but according to some authors it can be extended to 20 days. The patient becomes infectious from the last 1-2 days of the incubation period and within 7-14 days

Corresponding Author:

Dr. Bannova Svetlana Leonidovna

Federal State Budgetary Educational Institution of Higher Education "St. Petersburg State Pediatric Medical University" of the Ministry of

Health of the Russian Federation.

Email: svetlanalb81@mail.ru 
of the disease beginning (maximum infectiousness during the first 7 days). It is proved by a number of authors that in children the virus is excreted longer with faeces than from the upper respiratory tract (up to 28 against 14 days) [9]. COVID-19 in children, as well as in adults, clinically occurs as an acute respiratory viral infection (affecting only the upper respiratory tract), pneumonia without respiratory failure, pneumonia with acute respiratory insufficiency, acute respiratory distress syndrome, sepsis, and septic (infectious toxic) shock. About $45 \%$ of cases are asymptomatic [1-2-3-5-10].

In terms of gravity, COVID-19 flows in light, medium, heavy and extremely heavy forms [1-2-11]. The asymptomatic form is diagnosed in sick children in the absence of clinical signs of disease and visual changes on the X-ray (tomogram), but with a positive result of laboratory examination for SARS-CoV-2 RNA. Presence of minimal clinical signs as an increase in body temperature up to $38.50 \mathrm{C}$, cough, weakness, sore throat, as well as gastrointestinal manifestations such as nausea, vomiting, abdominal pain, diarrhea, in the absence of auscultatory changes in the lungs is typical for the mild form. It should be noted that gastrointestinal syndrome is much more common in children than in adults.

In the medium severe form the fever becomes higher than $38.50 \mathrm{C}$, dry unproductive cough, increased frequency of respiratory movements (FRM), shortness of breath only at physical activity is observed; auscultation of dry and moist wheezing, with no signs of respiratory insufficiency, pneumonia confirmed by CT of lungs, $\mathrm{SpO} 2<95 \%$, serum DRB more than $10 \mathrm{mg} / 1$ [1-2]. Signs of acute respiratory infection, progressing during the week, with respiratory failure, shortness of breath (FRM more than 30/min), $\mathrm{SpO} 2 \leq 92 \%$ are observed in severe form. On X-ray or chest CT scan the progression of pneumonia (increase in the area of infiltration changes by more than $50 \%$ in 24-48 hours), consciousness impairment, unstable hemodynamics (systolic BP less than $90 \mathrm{~mm} \mathrm{Hg}$ or diastolic BP less than $60 \mathrm{~mm} \mathrm{Hg}$, diuresis less than 20 $\mathrm{ml} /$ hour) [1-2-3].

Extremely heavy current is characterized by rapidly progressing disease with the development of acute respiratory distress syndrome (ARDS) and acute respiratory insufficiency (invasive ventilation of the lungs), septic shock, and multi-organ failure [1-2]. The indications for transfer of patients to ICU an increase in heart rate by more than $15 \%$ of physiological age at rest; cyanosis (in the absence of "blue" CHD) and shortness of breath, determined by visual examination, inflating the wings of the nose in children in the first year of life; hemoglobin saturation with oxygen from pulsating blood against the background of atmospheric air breathing $90 \%$ and below or, at donation of 1-2 1/ min of additional oxygen, - $93 \%$ and below; hypercapnia (pSO2 > $50 \mathrm{~mm} \mathrm{Hg}$ ). t.); decompensated acidosis $(\mathrm{pH}<$ 7,25); arterial hypotension with clinical manifestations of shock (limb marbling, acrocyanosis, cold limbs, capillary filling time of $4 \mathrm{sec}$ and more); lactate-acidosis (lactate concentration over $3 \% \mu \mathrm{mol} / 1)$; reduction of diuresis to oliguria level and below (below $50 \%$ of age norm and below); appearance of cough with blood admixture in sputum, chest pain or heaviness; appearance of signs of hemorrhagic syndrome.

In comparative analysis, according to various authors, the frequency of symptoms characteristic of respiratory disease in children and adults was found to be higher in children than in adults (58.5\% vs. $9.9 \%$ respectively). In contrast, hyperthermia is more common in adults. Such a symptom as weakness is rarely observed in children. More typical signs of acute respiratory disease, such as rhinitis, sore throat, hyperemia and granularity of the posterior pharyngeal wall, dyspnea, are common in children. Gastrointestinal vomiting and diarrhoea syndrome is also more common in children than in adults. In children currently in Russia, according to the recommendations of the Russian Ministry of Health, the following definition of a standard case should be applied:

- "Suspicious" case - the presence of clinical manifestations of acute respiratory infection, bronchitis, pneumonia, ARDS, sepsis, combined with the following data of epidemiological history (return from a foreign trip 14 days before symptoms appear; close contacts in the last 14 days with persons under observation for an infection caused by SARS-CoV-2, who subsequently became ill; close contacts in the last 14 days with persons who were laboratory-confirmed for COVID-19).

- A "confirmed" case is a positive result of laboratory testing for SARS-CoV-2 RNA by PCR regardless of clinical manifestations.

- The suspected diagnosis of COVID-19 neonatal infection is the presence of at least one clinical symptom, including unstable body temperature, low activity or poor nutrition, or shortness of breath; changes in the chest X-ray of the "frosted glass" type; presence among family members or caregivers of people with confirmed COVID-19 infection or close contact with people with confirmed COVID-19 infection or patients with severe pneumonia.

Indications for hospitalization of children with COVID-19 or suspicion of it are: severe or moderate severity of respiratory illness or non-hospital pneumonia; fever above $38.5{ }^{\circ} \mathrm{C}$, including according to medical history, or below $36.0^{\circ} \mathrm{C}$ or with fever duration above $38.0{ }^{\circ} \mathrm{C}$ for more than 5 days; shortness of breath or anxiety; tachypnea, unrelated to the fever, more than $20 \%$ of the age limit: Up to 1 year - more than 50 , from 1 to 5 years - more than 40 , over 5 years - more than 30 in min; tachycardia, not related to the fever, more than $20 \%$ of the age norm: up to 1 year - more than 140 , from 1 to 5 years - more than 130, over 5 years - more than 120 per min; partial oxygen pressure (in the presence of pulse oximeter) - $\mathrm{SpO} 2 \leq 95 \%$; depression of consciousness (drowsiness) or increased excitement, sleep inversion, refusal of food and drink; seizures; absence of positive dynamics or increase in clinical symptomatology in the background of the therapy 5 days after the disease starts; presence of severe background diseases (congenital and acquired heart diseases, including in the history (heart defects, rhythm disorders, myocardiopathy or myocarditis; chronic lung diseases (bronchopulmonary dysplasia, 
bronchial asthma, cystic fibrosis, bronchoectatic disease, congenital lung diseases, etc.; primary or secondary immunodeficiency, including HIV infection, autoimmune diseases, immunosuppressive therapy; oncohematological diseases, chemotherapy; metabolic diseases (diabetes mellitus, obesity, etc.); liver and kidney diseases); the impossibility of isolation when living with persons from risk groups; lack of conditions for treatment at home or guarantees of implementation of recommendations (hostel, social security institutions, temporary accommodation, socially disadvantaged family, poor social and domestic conditions).

The main method of SARSCoV-2 examination is PCR [1,2]. The main type of biomaterial for this study is nasopharyngeal and/or oropharyngeal smears. The examination is mandatory for the following categories of individuals:

- those who have come back to the Russian Federation with signs of respiratory diseases,

- those who have been in contact with a COVID-19 patient,

- those diagnosed with " non-hospital pneumonia",

- those over 65 years of age with symptoms of respiratory disease,

- medical workers who have the risk of COVID-19 infection at work (once a week, and when symptoms appear, not excluding COVID-19 - immediately),

- being in institutions of permanent residence regardless of the legal form of organization (special educational institutions of closed type, cadet corps, boarding houses, institutions of the Federal Service for the Supervision of Social Security of Russia) and the staff of such organizations - in case of symptoms of respiratory disease.

In addition, laboratory diagnostics includes clinical blood tests (leukopenia and lymphopenia), extended biochemical blood tests with obligatory examination of the level of CRP, coagulogram, smear testing for other respiratory viruses and, if necessary, screening for Streptococcus pneumoniae, Haemophilus influenzae type B, Legionella pneumophila by culture and/or PCR or rapid method, and for mycoplasma and chlamydia infections $[1,2,3]$. Radiation-based diagnostic techniques, such as CT, X-ray and chest ultrasound, are available to all patients with clinical signs of acute respiratory disease $[1,2,12]$. Focal changes may be detected on chest X-rays and in the absence of coughing. However, CT is preferred, with bilateral lung lesions occurring in children under 3 years of age, and unilateral lesions in children over 6 years of age $[2,11,13]$. A two-sided lesion and a high rate of change are the most characteristic of a severe illness $[2,10,14]$.

In order to provide prompt and high-quality medical care, the routing of patients with COVID-19 has been developed: - Patients with suspected COVID-19 and patients with mild disease (with no special indications for hospitalization) are isolated and treated at home. - Patients with suspected COVID-19 are isolated at home depending on their condition. - Patients with medium and severe illness are hospitalized in a specialized infectious disease hospital (for the treatment of patients with COVID-19). - In cases where COVID-19 infection is confirmed in the non-core ward, transfer to a specialized infectious diseases ward is made. - Patients with respiratory insufficiency II and more, severe pneumonia, critical conditions should be transferred to ICU immediately.

The general principles of therapy include bed rest, calorie adequate nutrition and adequate hydration, electrolyte balance and homeostasis control, monitoring of vital functions and oxygen saturation, control of respiratory tract permeability (according to indications - oxygen therapy), control blood and urine tests, blood gas analysis, repeated lung X-ray At present, children with COVID-19 have no unequivocal recommendations for the use of antiviral drugs due to lack of evidence. According to the recommendations of the Ministry of Health of Russia, the prescription of antiviral drugs should be individual, justified on a collegial basis. involving an infectionist and a pediatrician. The prescription of antiviral etiotropic therapy should take into account the age of the child, the presence or absence of concomitant pathology, the degree of severity of the disease, as well as possible drug interactions and contraindications [2].

As etiotropic (antiviral) therapy in children in Russia, the choice is recombinant interferon alpha- $2 b$ for intrapartum administration (in the form of drops and gel) and rectal administration. Interferon-alpha allows reducing the viral load at the initial stages of the disease, alleviating symptoms and reducing the duration of the disease, as well as viral excretion with faeces (in rectal use of interferon alpha with antioxidants) [2]. If recombinant interferon alpha-2b is contraindicated, Umifenovir is the alternative. Oseltamivir is administered in children only if it is confirmed to be associated with influenza. In the asymptomatic form of COVID-19, etiotropic therapy is not required in children. In the mild form of COVID-19 (ODS, non-symptomatic pneumonia), recombinant interferon alpha-2b (intra- or rectal) is the choice, the alternative is umifenovir [2].

In case of the medium severe form of COVID-19 (pneumonia with respiratory failure) with no severe concomitant diseases against the background of symptomatic therapy, recombinant interferon alpha$2 \mathrm{~b}$ (intra- or rectal) is recommended for children; the alternative is umifenovir. In children at risk it is recommended to prescribe such drugs as lopinavir/ ritonavir, hydroxychloroquine. In children with severe or critical COVID-19 form, hydroxychloroquine or a combination of hydroxychloroquine with tocilisumab or lopinavir/ritonavir or tocilisumab and lopinavir/ ritonavir is recommended. The prescription of systemic glucocorticosteroids is also shown. When bacterial complications progress, the use of intravenous immunoglobulins should be treated with extreme caution. In children aged 15 years and older the tactics of etiotropic drugs prescription recommended for adults may be considered.

Children with a fever above $38.5^{\circ} \mathrm{C}$, as antipyretic agents use ibuprofen or paracetamol (inside or in the form of rectal suppositories) in the age dose, as well as possible 
to use physical methods of cooling. Acetylsalicylic acid and nimesulide are not used in children with antipyretic purposes. Metamisol should not be used because of the high risk of agranulocytosis.

Anti-coughing, expectorant, mucolytics, including numerous patented preparations with various herbal products, are not recommended for routine use due to inefficiency. The use of mucolytic and expectorant agents (ambroxol, acetylcysteine, carbocysteine) is recommended only for viscous, difficult to separate sputum.

In the presence of bronchial obstruction syndrome, it is possible to use bronchodilators (salbutamol or a combination of fenoterol and ipratropium bromide) in standard doses, preferably the use of dosed inhalers through a spaceer. For patients with bronchial asthma, the basic therapy is the same.

Enteral fluid intake in the volume of age requirement is shown to prevent dehydration.

The discharge of children with laboratory confirmed diagnosis of a new coronavirus infection is allowed in case of absence of clinical manifestations of the disease and obtaining a double negative result of laboratory examination for SARS-CoV-2 RNA at an interval of at least 1 day [1-2-3].

\section{References}

1. Temporary guidelines for the prevention, diagnosis and treatment of new coronavirus infection (COVID-19) (version 5) 04/02/2020, Moscow. 2020.

2. COVID-19. Temporary guidelines (version 1 ) of $04 / 03 / 2020$, Moscow. 2020;:-68 p.

3. A collection of guidelines, algorithms for the actions of medical workers at various stages of care, checklists and standard documents developed for the period of presence and threat of further spread of a new coronavirus infection in St. Petersburg. Version 1.0 dated 04.17.2020, St. Petersburg. $2020 ;: 157 \mathrm{p}$.

4. To KK, Tsang OT, Leung W, Tam AR, Wu T, Lung DC, Yip CC, Cai J, Chan JM, Chik TS, Lau DP, Choi CY, Chen L, Chan W, Chan K, Ip JD, Ng AC, Poon RW, Luo C, Cheng VC, Chan JF, Hung IF, Chen Z, Chen H, Yuen K. Temporal profiles of viral load in posterior oropharyngeal saliva samples and serum antibody responses during infection by SARS-CoV-2: an observational cohort study. The Lancet Infectious Diseases. 2020 05;20(5):565-574. https://doi. org/10.1016/s1473-3099(20)30196-1

5. Ong SWX, Tan YK, Chia PY, Lee TH, Ng OT, Wong MSY, Marimuthu K. Air, Surface Environmental, and Personal Protective Equipment Contamination by Severe Acute Respiratory Syndrome Coronavirus 2 (SARS-CoV-2) From a Symptomatic Patient. JAMA. 202004 28;323(16):1610. https://doi.org/10.1001/jama.2020.3227

6. Guidelines organization of medical care for pregnant women, women in labor, women in childbirth and newborns with a new coronavirus infection COVID-19, version 1 (04.24.2020) Moscow:61 p.

7. Dong $\mathrm{Y}$, Mo X, Hu Y, Qi X, Jiang F, Jiang Z, Tong S. Epidemiology of COVID-19 Among Children in China. Pediatrics. 202003 16;145(6):e20200702. https://doi. org/10.1542/peds.2020-0702

8. Brodin P. Why is COVID-19 so mild in children?. Acta
Paediatrica. 202004 15;109(6):1082-1083. https://doi. org/10.1111/apa.15271

9. Guidelines features of clinical manifestations and treatment of the disease caused by a new coronavirus infection (COVID-19), version 1 (04.24.2020) Moscow, - 44 p..

10. Wenjie Tan, at al. Notes from the Field: A Novel Coronavirus Genome Identified in a Cluster of Pneumonia Cases - Wuhan, China 2019-2020. China CDC Weekly. 2020;2(4):61-2.

11. Lu X, Zhang L, Du H, Zhang J, Li YY, Qu J, Zhang W, Wang Y, Bao S, Li Y, Wu C, Liu H, Liu D, Shao J, Peng X, Yang Y, Liu Z, Xiang Y, Zhang F, Silva RM, Pinkerton KE, Shen K, Xiao H, Xu S, Wong GW. SARS-CoV-2 Infection in Children. New England Journal of Medicine. 202004 23;382(17):1663-1665. https://doi.org/10.1056/ nejmc 2005073

12. Xu Y, Li X, Zhu B, Liang H, Fang C, Gong Y, Guo Q, Sun X, Zhao D, Shen J, Zhang H, Liu H, Xia H, Tang J, Zhang K, Gong S. Characteristics of pediatric SARS-CoV-2 infection and potential evidence for persistent fecal viral shedding. Nature Medicine. 202003 13;26(4):502-505. https://doi. org/10.1038/s41591-020-0817-4

13. Zheng F, Liao C, Fan Q, Chen H, Zhao X, Xie Z, Li X, Chen C, Lu X, Liu Z, Lu W, Chen C, Jiao R, Zhang A, Wang J, Ding X, Zeng Y, Cheng L, Huang Q, Wu J, Luo X, Wang Z, Zhong Y, Bai Y, Wu X, Jin R. Clinical Characteristics of Children with Coronavirus Disease 2019 in Hubei, China. Current Medical Science. 2020 03 24;40(2):275-280. https:// doi.org/10.1007/s11596-020-2172-6

14. Sun D, Li H, Lu X, Xiao H, Ren J, Zhang F, Liu Z. Clinical features of severe pediatric patients with coronavirus disease 2019 in Wuhan: a single center's observational study. World Journal of Pediatrics. 202003 19;16(3):251-259. https://doi. org/10.1007/s12519-020-00354-4

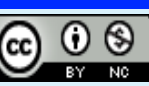

This work is licensed under a Creative Commons AttributionNon Commercial 4.0 International License. 